\title{
A Comparison of On-premise to Cloud Migration Approaches
}

\author{
Claus Pahl, Huanhuan Xiong, Ray Walshe \\ IC4, Dublin City University \\ Dublin 9, Ireland \\ http://www.ic4.ie/
}

\begin{abstract}
While cloud computing has certainly gained attention, the potential for increased uptake of the technology is still large. As a consequence, how to move and migrate to the cloud is an unanswered question for many organisations. Gaining an understanding of cloud migration processes from on-premise architectures is our aim here. For this purpose, we look at three provider-driven case studies based on the common three layers of cloud computing: Infrastructure (IaaS), platform (PaaS) and software (SaaS) as a service. These shall be complemented by a fourth, independent systems integration perspective. We extract common migration process activities for the layer-specific processes and discuss commonalities, differences and open issues. The results presented are based on expert interviews and focus groups held with major international cloud solution providers and independent consultants.
\end{abstract}

Keywords: Cloud Migration; Cloud Migration Processes; On-premise to Cloud; Cloud Architecture.

\section{Introduction}

Software application hosting settings range from on-premise solutions to private clouds to public clouds [1]. The migration into the cloud or between these often hybrid scenarios or between offerings is a key concern and the determination and assessment of possible migration processes is important. We carried out research into migration scenarios in the form of migrations processes and patterns based on expert interviews and focus groups with major international cloud solution providers and independent consultants. The proposed processes document a range of different architectural settings. They highlight the concerns - like costs, skills and technologies - to be considered to deploy applications in various cloud scenarios and to migrate into these from on-premise architectures. Attention also needs to be given to hybrid solutions where parts of an application system remain on-premise and parts are migrated to the cloud, maintaining an efficient division of responsibilities and effective data flows.

In order to start the migration process determination and evaluation, an empirical investigation into migration processes was conducted at the Irish Centre 
for Cloud Computing and Commerce (IC4). A structured methodological approach was followed, involving IC4 industry consortium members (http: \\www . ic4.ie) with background or experience in cloud migration. We conducted expert interviews to gain a broader understanding beyond individual cases than would have been possible through concrete projects observation alone. The experts would have had 15-20 years of industrial practice and a minimum of 3 years in cloud migration.

Our work has focused on determining the principle cloud migration processes. Our investigation has shown differences between the cloud deployment models IaaS, PaaS and SaaS, which we will reflect by model-specific migration processes based on a catalogue of common activities. We emphasise the differences and commonalities between the three layers, but also use an independent broker acting as a systems integrator (in contrast to the other more provider-based migration) to broaden the view and evaluate previous results.

We discuss some foundations for our study in Section 2, before detailing the results in Section 3. The observations are discussed in Section 4, before summarising related work in Section 5 .

\section{Assumptions and Background}

The migration layers that we identified are mainly derived from the user-oriented service models SaaS, PaaS and IaaS $[1,10]$. The concerns shall briefly be outlined:

- SaaS. Companies look for a migration solution to move their existing onpremises applications to a cloud environment. Application vendors frequently want to evaluate a cloud platform on which to deploy a new application or SaaS offering.

- PaaS. PaaS Migration is the process of moving from the use of one software operating and deployment environment to another environment. At this layer customers do not manage their virtual machines, but rely on the infrastructure layer's compute and storage resources. They merely create applications within an existing API or programing language.

- IaaS. IaaS migration is mainly offering virtual machines as a (compute) service to users, such as moving from one VM to another, or managing or interoperating the different VMs. In addition, storage or network capabilities can also be provided. Instead of purchasing servers or even hosted services, IaaS customers can procure and operate servers, data storage systems, or networking resources at will.

We define cloud migration as follows. Cloud migration is the process of partially or completely deploying an organization's digital assets, services, IT resources or applications to the cloud. The cloud migration process may involve retaining some IT infrastructure on-site. In such a scenario, the existing system may be fused with a partial cloud solution that can be hosted by a third party over the Internet for a fee. The cloud component of this hybridised system can transition between several cloud providers allowing businesses to choose the 
most cost-effective solution. However, the migration process involves the risk of accidentally exposing sensitive business critical information. Therefore, cloud migration requires careful analysis, planning and execution to ensure the cloud solution's compatibility with organizational requirements, while maintaining the availability and integrity of the organization's IT systems.

Our motivation is to determine common migration processes and decompose them into operational level activities in order to make the migration process more transparent, agile, and suitable for a variety of business models. Our research will provide an understanding of the core mechanism to assist SMEs (Small to Medium Enterprises) in particular with the migration of their IT infrastructure to the Cloud. We will concentrate particularly on the area of managing the transition of on-premises applications to the Cloud. Our ultimate aim (beyond the aims of this investigation here) is to develop a system to minimise the inherent complexities involved with the migration to a Cloud Computing environment.

\section{Cloud Migration Case Studies}

The three case studies SaaS, PaaS and IaaS, as well as the systems integrator case study shall now be looked at individually. The results presented here were extracted from interviews and focus groups held with migration experts. Primarily, on-premise to cloud migration was considered. A common understanding of cloud migration processes was assumed as follows:

A cloud migration process is a set of migration activities carried to support an end-to-end cloud migration. Cloud migration processes define a comprehensive perspective, capturing business and technical concerns. Stakeholder with different backgrounds are involved.

Thus, initial requirements and expectation elicitations are part of the processes as are tools for automated migration of IT artefacts or plans for the deployment of new cloud services and decommissioning of old infrastructure.

We define typically three or four top-level activities that are performed as discrete, sequenced steps. For each activity, we describe the following concerns:

\begin{tabular}{|l|l|}
\hline Concern & Concern of the Respective Activity \\
\hline \hline focus & $\begin{array}{l}\text { a differentiation whether the focus is technical or business- } \\
\text { oriented in nature }\end{array}$ \\
\hline from-to & source and target of migration activity \\
\hline $\begin{array}{l}\text { vision } \\
\text { ignorance }\end{array}$ & $\begin{array}{l}\text { a distinction of migration benefits amd expectations that po- } \\
\text { tential users are aware of (their 'vision') and those overlooked } \\
\text { (their 'ignorance') }\end{array}$ \\
\hline cases & an identification of any distinguishable special cases \\
\hline stakeholder & the stakeholder involved \\
\hline artefacts & the IT artefacts involved \\
\hline steps & the individual, smaller steps involved in the activity \\
\hline
\end{tabular}




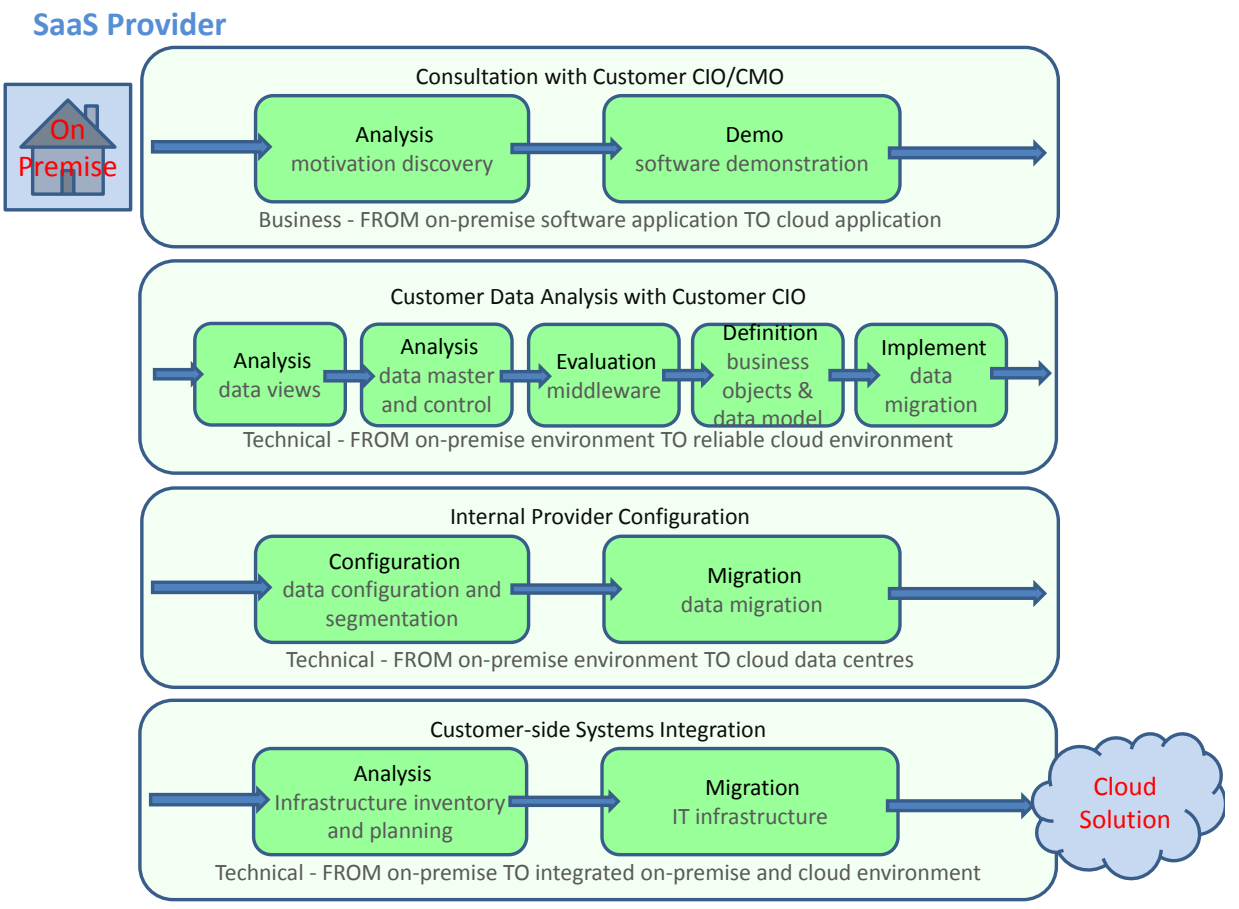

Fig. 1. SaaS Migration Processes.

The top-level process activities and the individual steps will be summarised in respective diagrams. The discussion reflects the perspective of the solution providers, e.g. their aim to clarify benefits, but also potential problems.

\subsection{SaaS-level Migration}

For SaaS-level migration (Fig. 1), the software applications under consideration here are classical ERP packages for accounting, HR management, CRM etc., but also domain-specific software for the retailing or engineering sector would apply.

\section{Consultation with Customer CIO (or CMO)}

- Focus: Business

- From-To: FROM on-premise software TO cloud software application Cloud application providers aim to sell their product, but also need to guide the process. An important element of the discussion with customers is to emphasise that a democratisation of software provisioning takes place, i.e. the same service is provided for everyone.

- Vision: more agility through a more declarative way of configuring and managing software and a drag \& drop style of operating applications. 
- Ignorance: cognitive dissidence, i.e. losing control, is often a concern. IT people do not like to lose systems they built. A more business-oriented concern is the licensing status that might vary from tool to tool, which is a financial issue.

The following steps can be identified:

- Step 1: motivation discovery (why would you do this) for small companies

- Step 2: software demonstration to address all actors concerns (meeting or phone call) as an additional step for midsize companies

\section{Customer Data Analysis with Customer CIO}

- Focus: Technical

- From-To: FROM on-premise environment TO reliable cloud environment Data transformation is usually the technical solution, but in terms of expected benefits, a reliable cloud environment is the driver. Failure assurance (failover), analysis and audit capabilities are much better in professionally managed cloud solutions. This requires a technical discussion under these headings as to what data would or should be moved, what views and responsibilities exist. A first analysis and selection at this stage will define who will master data and ascertain that for instance no garbage is migrated. A data loader can then be configured and used accordingly.

The following steps can be identified:

- Step 1: what view on data: 180-360 degree perspectives

- Step 2: who will master data

- Step 3: evaluate middleware solutions: once-off, incremental or (fullblown) integration in the cloud

- Step 4: the customer MIS plan defines the application data model (model the business object such as a customer, an account, activities)

- Step 5: select core data for migration: segmentation if a data model exists

\section{Internal Provider Configuration}

- Focus: Technical

- From-To: FROM on-premise environment TO cloud data centres

The concern is the provider-side (internal) configuration and segmentation of data for transfer and storage. SaaS providers often provide data migration tools through sales infrastructures like app marketplaces.

Two cases can be distinguished. SMBs (small-mid business) have a customisation need arising from the B2B environment where efficiency and growth are the drivers. USBs (ultra-small business) require configuration for their $\mathrm{B} 2 \mathrm{C}$ environment, targeting engagement, campaigns and loyalty as drivers.

\section{Customer-side Systems Integration}

- Focus: Technical

- From-To: FROM on-premise environment TO integrated on-premise and cloud environment

Rarely all on-premise IT infrastructure will be migrated into a cloud environment. Thus, an integration between different on-premise and (hybrid) cloud solutions is required. Larger solution providers offer these integration tools, the IBM Websphere Cast Iron cloud integration is an example. 
An often observed problem concerns IT staff: they often feel overstretched due to recent virtualisation cycles in their organisations and do not like their own systems to be abandoned. Cooperation can consequently be reluctant.

Migration should be more than just redoing past activities in the cloud, i.e. cloud migration creates an opportunity to transform architectures and internal processes to some extent.

Success of the migration process is of importance for both providers and their clients. This can be measured in terms of different metrics such as headcount (a business concern for the provider) or fewer servers in use (a technical concern for the client).

Like any other process, cloud migration benefits from tools and proven techniques to be applied. The data loader is a tool example for most application software. In addition, a list of questions alone or better a template for discussions with questions, particularly for the first stage, is useful. As some of the software applications are common in organisations, so are migration, integration and deployment support. There is a noticeable differentiation between organisations of different size, only offering standard solutions for smaller clients.

\subsection{PaaS-level Migration}

PaaS solutions provide support for the development and deployment of software. We will particularly focus on development (Fig. 2), with ISVs (independent software vendors) supporting other organisations with their development. This provides a more complex PaaS setting. Specifically, we consider here a PaaS solution by a multinational, providing a platform based on globally distributed datacentres. SDKs for different development languages are available.

\section{Consultation with ISV CEO}

- Focus: Business

- From-To: FROM classical licensing model TO SaaS

The transfer is often forced by an ISV's end customers. As the latter are the ISV's PaaS customers, as a consequence, a SaaS/PaaS alignment is required where the ISV solution is made available as a SaaS, making the situation more complex than the previous SaaS case. Also, the PaaS providers as multinationals are companies that often work with partners (consultancies), which can be involved at all levels.

- Vision: costs, sales and marketing are the drivers for an ISV to adopt cloud development. The value proposition is that hosting is outsourced, i.e. no more management of infrastructure is required.

- Ignorance: while in general benefits and concerns such as security or failure are clear (and would be covered in SLA negotiations), some major changes result as implications that are not fully understood. This includes changed cash flow from a reliable up-front licensing model to a more unreliable pay-as-you-go or post-usage billing. Another major aspect overlooked is a necessary skills change. This applies to IT managers in particular, e.g. in relation to security technology like firewalls, but also the developers themselves, as we will discuss later on. 


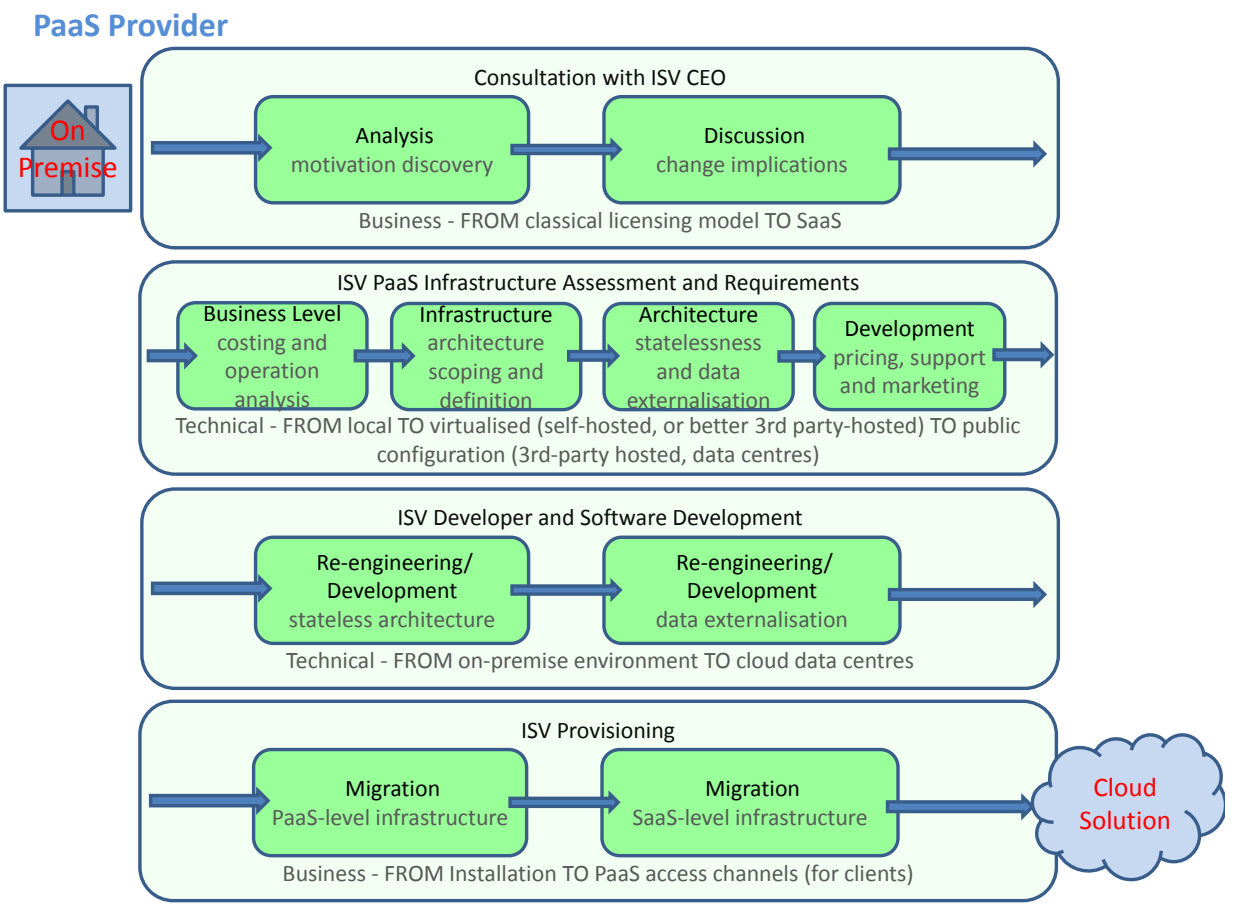

Fig. 2. PaaS Migration Processes.

\section{ISV PaaS Infrastructure Assessment and Requirements}

- Focus: Technical

- From-To: FROM local TO virtualised (self-hosted, or better 3rd partyhosted) TO public configuration (3rd-party hosted, data centres)

The technology focus is on determining the elements of the existing IT development infrastructure and any dependencies between the components. A dependency analysis (using tools where possible, e.g. scanning networks to detect dependencies) needs to be carried out - this includes applications and machines. Already virtualised solutions are easier to move.

\section{ISV Developer and Software Development}

- Focus: Technical

- From-To: FROM Traditional OO/SC/Server Architectures TO cloud PaaS architecture

Development using a PaaS environment requires a number of major changes regarding the architecture design and software development approach.

- Firstly, statelessness is a requirement for virtual machines (VMs) to be deployable without data.

- Secondly, as a consequence, data externalisation is required to prepare for scale-out, which necessitates externalisation for an efficient management of elasticity requirements. 
Consequently, this requires the developer to change development styles, possibly in a significant way depending on the current approach.

\section{ISV Provisioning}

- Focus: Business

- From-To: FROM Installation TO PaaS access channels (for clients)

The business focus reflects the transfer of cloud advantages from the ISV (at the PaaS-level) to its client (at the SaaS-level). Corresponding access channels to the new cloud deployment platform need to be provided.

As for SaaS, supporting techniques would be beneficial, but due to the increased complexity, these have less of an impact [7]. Commonly used are question catalogues (typical are 100 questions that help to capture current and envisioned development architectures) - used manually by PaaS provider consultants in the early stages of the migration process. Sometimes, these questions are organised into decision trees to guide and focus their application [6].

Some other observations are noteworthy. In contrast to the SaaS observations, the business side can be difficult to convince, while it is easier with IT staff. This indicates that changed cash flow is more of a problem than IT development reskilling. Despite this observation, the architecture complexity (Stage 3) is often underestimated.

Success criteria that are applied here are expenditure-based and end-customer numbers (of ISVs) as metrics.

\subsection{IaaS-level Migration}

At the IaaS level (Fig. 3), a number of different concerns including VM migration and big data migration emerge. We take a comprehensive view, i.e. migration of a full IT infrastructure is the setting.

\section{Business Case Determination}

- Focus: Business

- From-To: FROM on-premise installation TO IaaS solution

The key drivers are cost (reduction in operational expenditure is aimed at, but migration costs are often neglected), time (will almost always be incremental), impact (adding flexibility), strategy (what part of the operation or business would gain, which would suffer pain). Generally, given the normal scale, an incremental approach is taken. A testbed is defined and a migration strategy based on best-practice is selected where possible.

- Vision: the drivers listed above have summarised the main concern, but cost reduction and increased flexibility are important expectations.

- Ignorance: the 'pain versus gain' problem, i.e. negative aspects and the cost of required changes are sometimes neglected.

\section{Assessment and Planning}

- Focus: Business

- From-To: FROM on-premise installation TO IaaS solution 


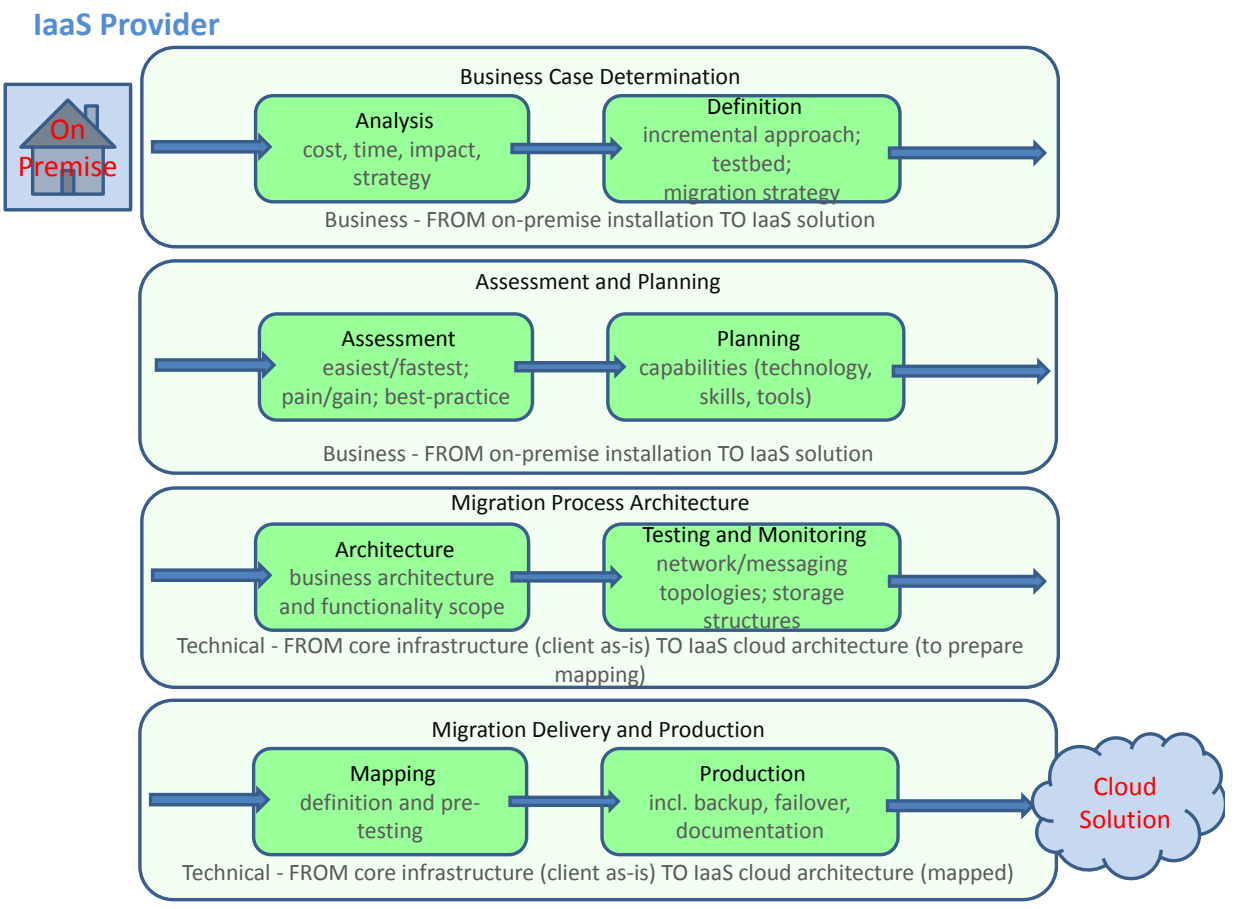

Fig. 3. IaaS Migration Processes.

Input for this assessment stage includes a determination of the 'easiest/fastest' aspects (in order to start an incremental process), the pain/gain flow chart (gains versus costs) and, if possible, best-practice/reference cases. As part of a contracted process, success criteria are also determined.

The planning involves the determination of the required capabilities for the migration in terms of technology, skills and tools. Specifically, this could involve assessing the IaaS provider's own capabilities, i.e. tools available, the need to bring in contract partners. This results in building a migration project team, bringing together provider and customer. The project planning defines milestones, metrics, and role distribution for the project team.

\section{Migration Process Architecture}

- Focus: Technical

- From-To: FROM core infrastructure (client as-is) TO IaaS cloud architecture (to prepare mapping)

The technical focus starts with the business architecture and defines the functionality scope for the cloud architecture. Testing and monitoring are aspects that need to be addressed at this stage. The architecture concerns here are network, directory structures, the messaging topology and the application topology based on the identified business functions - there should be a cloud solution for each function as a guideline. Generally, the client's 
development methodology and processes and well as the operations processes need to be adapted. This follows our observations for the PaaS layer.

4. Migration Delivery and Production

- Focus: Technical

- From-To: FROM core infrastructure (client as-is) TO IaaS cloud architecture (mapped)

The technical work includes the following steps: mapping definition and a pre-testing step before the new system is put into production. Despite some technical elements, the process is based on experience (past projects and general best-practice) are considered for migration projects as a whole. Production involves backup and documentation. Failover and other failure management need to be considered here as part of the migration of a production system.

- an incremental approach plugs in components individually into the customer architecture and tests them,

- a backup is to be kept both on-premise and in-cloud in operation while data are moved; only then can decommissioning of on-premise infrastructure start. Decommissioning needs a planned approach.

Proper documentation and specification is, of course, a key concern.

Success criteria that can be applied here are the time frame or the metrics (by milestone) as discussed earlier.

Tool support is, as with the other cases, varied. On a higher level, reference case and best-practice approaches play an important role. Only in the technical context of virtualization technology, tools and automation are available. We can easily create virtual machines consisting of virtualized processor, communications, storage, networking, and I/O resources. Standards like OVF for VM packaging and exchange and OCCI as an example for VM lifecycle management or TOSCA for portability at the IaaS layer (and compliant tools) can be utilised.

Again, architecture emerges as critical concern. Specific to this level, networks, storage and messaging and application topology are the aspects.

\subsection{Systems Integrator}

This case study (Fig. 4) has a validating role as a number concerns already addressed above will reappear here. Again, the focus is on-premise to cloud migration. The company providing input for our study has acted as an intermediary/consultant supporting others to use a cloud service and as a service solution provider (another SaaS example), for instance an ISV-SaaS provider of accounting systems using a PaaS platform like Google Apps. Systems integration refers to the utilisation of infrastructure technologies to communication and processes to be supported [18].

1. Customer - Business Analysis As in other cases, the main expected benefit is cost reduction. Another confirmation applies to the PaaS model. Cash flows change, but equally important, there is no hybrid between a software licence model and a SaaS model. A clear decision needs to be taken. 
Independent Consultant / Systems Integrator

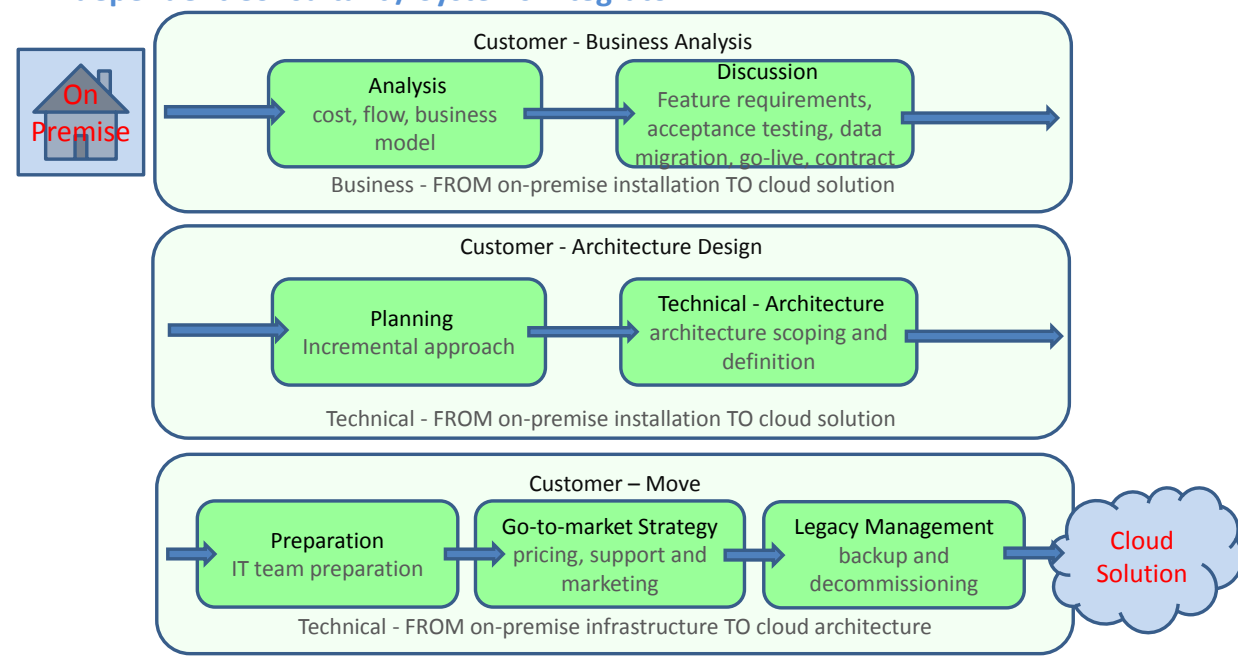

Fig. 4. Systems Integrator Migration Processes.

The initial consultation with the client involves a discussion covering the following aspects and later steps of a migration process (a sample system type would be an accounting system moved to the cloud):

- Feature requirements determination

- User acceptance testing

- Data migration

- Go-live discussion

- Contract discussion

2. Customer - Architecture Design The determination of the architectural scope is the aim. As in other complex cases, an incremental execution is the preferred solution. A lean startup solution would start with the top 5 out of 50 features as the first step, adding features in regular extensions. For a consultant, platform (and provider) selection is the first major decision.

- At the SaaS layer, e.g. Salesforce is a leading provider of CRM software. The ecosystem can play a role here. Salesforce's App Exchange on top of its CRM provides additional benefits in terms of migration support and systems integration.

- At the PaaS layer, e.g. MS Azure supports a variety of development languages such as Python and, of course, the .NET platform. However, Microsoft is less advanced in terms of marketplace solutions.

Another dimension in choosing a provider are the different product lines, such as premium, standard and free, offering choice to the client.

3. Customer - Move The final stage addresses business and technical concerns. This go-to-market stage needs an IT team preparation activity.

Architecture emerges as a critical concern, what we have already noted. Architecture problems do occur and improperly architected solutions need to 
be targeted. There is often a need (but also an opportunity) to redesign a cloud architecture solution (e.g. storage costs are often underestimated, which either requires unnecessary garbage data to be removed or a differently configured solution to be used). Possibilities for configuration that cloud solutions offer are often, at least initially, not considered. Changes in the cloud solution architecture would anyway require an agile approach to architectures.

Legacy management is another concern that needs to be looked at from the outset. Decommissioning needs to take place (as discussed for the IaaS layer). Backup systems are useful at early migration stages, but provide consistency problems later on and incur costs.

\section{Analysis}

A summary of the processes employed by the four different migration solution provider with their essential activities is given in Figure 5 .

Our vision and ignorance discussion has demonstrated a good understanding of the benefits, but also that a number of concerns emerge that potential users and also providers do not properly understand and address - both business and technology issues.

- Technical: As a critical issue, the central role of the architecture emerges [19]. Stateless components and data externalisation are required if cloud advantages like elasticity are to be gained. Consequently, re-architecting is often necessary if more than data transfer into the cloud (for standard applications) is the migration scope.

- Business: Skills and cash flow emerge as two concerns. Particularly, for some IT staff, more emphasis needs to be put on integration, configuration and security. A cloud solution will not only facilitate the change from capital expenditure to operational expenditure as a positive aspect, but create different cash flow situations for users of infrastructure or platform solution who themselves become cloud solution providers.

The attitude of stakeholders varies, e.g. IT specialists can react in a positive, but also negative way to cloud migration plans. An understanding of the technical benefits exists, but can be counteracted by the fear to lose control and status as software is created and managed elsewhere.

Security, trust and data protection are all-encompassing issues that we have not covered here, due to their very different technical nature compared to the architecture concerns.

While we acknowledge that the processes presented here are limited to specific solution providers and might be not be considered as generalised, we have tried to alleviate this concern by specifically selecting experts with a long experience (often across companies) and by using the results obtained from the independent consultant to validate the other three case study results. 


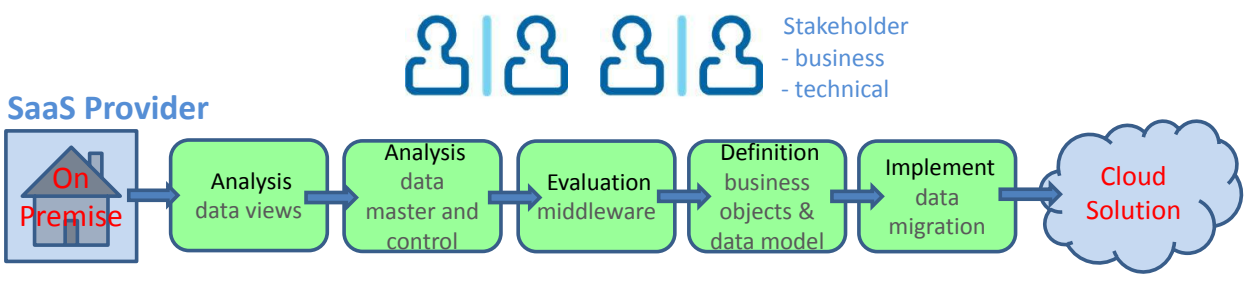

PaaS Provider

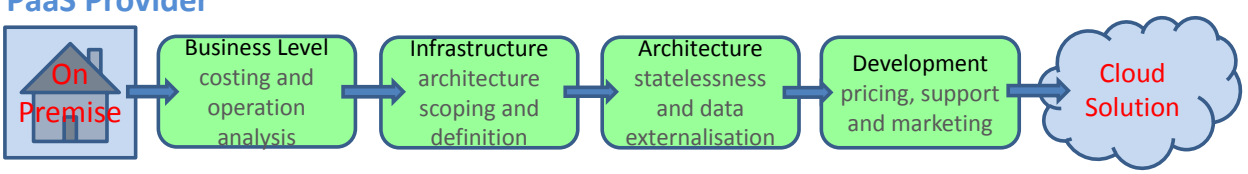

laaS Provider

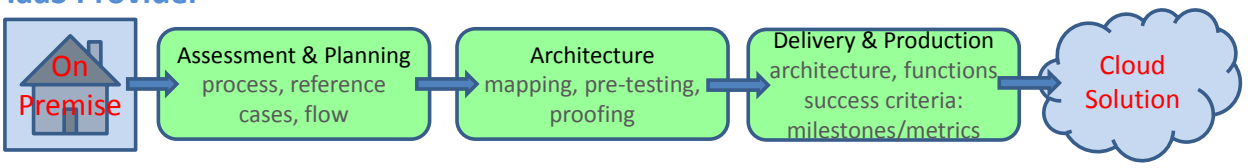

Independent Consultant / Systems Integrator

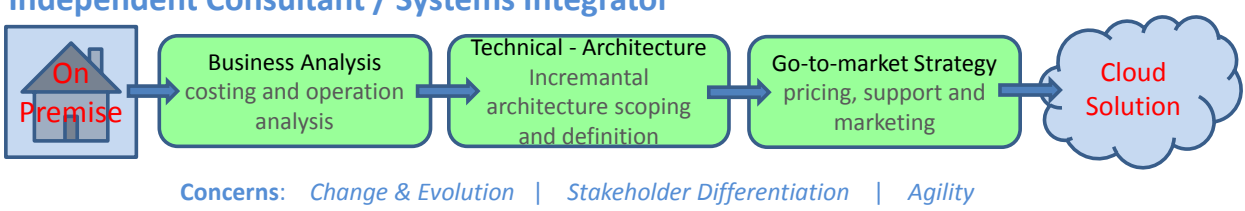

Fig. 5. Migration Processes Framework - Use Cases and Selected Activities.

\section{Related Work}

Related research focuses for instance on an advanced model-driven methodology and tools for reuse and migration of on-premises applications to cloud. To support the migration, e.g. the REMICS project (EU FP7) enhances the OMG Architecture Driven Modernization (ADM) methodology with specific methods, meta-models and tool support, including knowledge discovery, patterns and transformations for SOA and Cloud Computing.

The REMICS project is looking at a model-driven solution to support migration [19-21]. Part of the work concentrates on the opportunity for software modernisation - a challenge, but also opportunity that we have also identified. Their architecture-driven modernisation extracts the legacy architecture in order for it to form the basis of the new cloud solution. Automated translation of business process, component and test specification preserves existing features, and will also allow weaving in new architectural elements in a coherent way. The SMART process governs the sequence of activities. However, our concern here was less to provide a concrete software architecture solution - rather to look at concerns from a broader management perspective. 
A number of practical guidelines exist, published by cloud solution providers to aid the migration to their own products:

- Amazon provides whitepapers for its Amazon Web services solutions (including e.g. EC2) [14]. These AWS whitepapers outline the target architecture, their interfaces and also migration aspects.

- Similar documents are published by other companies, such as IBM [5], Salesforce [4] or Oracle [11,12].

- Microsoft as another major provider in the PaaS space also provides whitepapers [2]. Additionally, technology evangelists like David Chappell provide material in various media types [3].

Data migration [9] emerges as a more mature migration concern. An aspect that can be tool-supported is cost estimation [8], which can alleviate initial concerns.

\section{Conclusions}

Migration to the cloud raises currently a range of questions. Common procedures do not exist and tool support is often not available. Migration experts rely on their own experience and some basic tools to facilitate the process. We have aimed to identify commonalities in the migration process in different context, using the cloud IaaS/PaaS/SaaS layers as the primary differentiation factor.

We have established core elements of a migration process toolkit like standard activities and steps, based on facets of (a here implicit) cloud migration ontology defining major concerns. The activities and steps across the different layers point to a common set of migration tasks that can be assembled to match the needs of the different deployment layers, but also provider and user types. The discussion has highlighted the immaturity in terms of established procedures and availability of tools to support the process. Important challenges arising from our observations include the importance of adequate architecture design for the cloud, but also the implications in terms of changed business models.

A plan arising from this discussion is a migration pattern catalogue. Patterns are templates that can be applied in a concrete situation. A migration pattern would be more specific than the processes described. In addition to cloud layers, which we have used to discriminate between different cloud migrations, a number of other factors arise from our discussion. The size of the organisation plays a role. It dictates the needs, but also the financial scope, which in turn limits the effort a provider will make to accommodate special configurations. Distinguishing between different SaaS application categories (e.g. ERP versus technical software) makes sense as well as distinguishing more clearly between PaaS development and deployment or IaaS compute, storage and networking.

\section{Acknowledgements}

The authors are greatly indebted to the participants of the IC4 migration studies. 


\section{References}

1. Mell, P. and Grance, T.: The NIST Definition of Cloud Computing. NIST Special Publication 800-145 (2010)

2. Server and Cloud Platform, http://www.microsoft.com/en-us/servercloud/default.aspx (2013)

3. Chappell, D.: How SaaS Changes an ISV's Business, Sponsored by Microsoft Corporation. http://www.davidchappell.com/writing/white_papers/ How_SaaS_Changes_an_ISVs_Business-Chappell_v1.0.pdf (2012)

4. Developerforce, http://wiki.developerforce.com/page/Tools (2013)

5. IBM: Cloud Computing Reference Architecture 2.0. https://www.ibm.com/developerworks/mydeveloperworks/blogs/c2028fdc-41fe4493-8257-33a59069fa04/entry/chapter_13_cloud_computing_reference_architecture1 ?lang $=$ en $(2011)$

6. Skilton, M., Gordon, P.: Cloud Buyers' Decision Tree. The Open Group (2010)

7. In, H.: Conflict Identification and Resolution for Software Attribute Requirements. PhD dissertation. University of Southern California (1998)

8. RightScale: PlanForCloud. http://www.planforcloud.com/ (2012)

9. Mohanty, S.: Data Migration Strategy. http://www.informationmanagement.com/specialreports/20040518/1003611-1.html (2004)

10. Murtaza, S., Masud, R.A.: An Extended and Granular Classification of Cloud's Taxonomy and Services. International Journal of Soft Computing and Engineering 2(2), 278-286 (2012)

11. Oracle: SQL Developer Migration. http://www.oracle.com/technetwork/ products/migration/sqldevelopermigration21-wp-131240.pdf (2010)

12. Oracle: After Migrating or Upgrading the Database. http://docs.oracle.com/ cd/A87860_01/doc/server.817/a86632/migaftrm.htm (2011)

13. Rational Software Development Platform: Migrate from VisualAge Generator 4.5 to the Enterprise Generation Language (EGL). IBM. http://www.ibm.com/developerworks/rational/library/egldoc.html (2012)

14. Varia, J. Migrating your Existing Applications to the AWS Cloud: A Phasedriven Approach to Cloud Migration, AWS Cloud Computing Whitepapers, http://media.amazonwebservices.com/CloudMigration-main.pdf (2010)

15. Email Migration Toolkit, http://www.sitaas.de/fileadmin/data/ Email_Migration_Toolkit.pdf (2013)

16. Pahl, C., Giesecke, S., Hasselbring, W.: Ontology-based modelling of architectural styles. Information and Software Technology 51 (12), 1739-1749 (2009)

17. Wang, M.X., Bandara, K.Y., Pahl, C.: Process as a Service Distributed Multitenant Policy-Based Process Runtime Governance. IEEE International Conference onServices Computing (SCC), pp. 578-585. IEEE (2010)

18. Halvey, J.K., Melby, B.M.: Business Process Outsourcing: Processes, Strategies, and Contracts. John Wiley \& Sons, Inc., Hoboken, New Jersey (2007)

19. Mohagheghi P., Saether, T.: Software Engineering Challenges for Migration to the Service Cloud Paradigm. World Congress on Services'11, pp. 507-514. IEEE (2011)

20. Mohagheghi, P., Berre, A.J., Sadovykh, A., Barbier, F., Benguria, G.: Reuse and Migration of Legacy Systems to Interoperable Cloud Services - The REMICS project. Mda4ServiceCloud'10 Workshop (2010)

21. Orue-Echevarria, L., Alonso, J., Escalante, M., Benguria, G.: Moving to SaaS: Building a migration strategy from concept to deployment. In: Ionita, A.D., Litoiu, M., Lewis, G. (eds) Migrating Legacy Applications: Challenges in Service Oriented Architecture and Cloud Computing Environments. IGI (2012) 